\title{
Logistics research trends 2012: a mid-year snapshot
}

\author{
Peter Klaus
}

Published online: 3 July 2012

(C) Springer-Verlag 2012

In early June of this year two significant logistics research conferences to place in Europe: The 24th annual Nordic Logistics Research Network Conference "NOFOMA 2012" at Turku, Finland, and the 6th International Scientific Symposium on Logistics by BVL, the international German logistics association at Hamburg, Germany. In Turku an impressive range of more than 80 presentations were given to an audience of 130 professors, senior researchers and doctoral candidates. At Hamburg 26 full research based papers were discussed to another 150 participants from academia and industry.

Presentations at academic conferences offer an excellent opportunity to get an up-to-date impression of current research trends, because many papers are still "work-inprocess". Question-and-answer sessions following presentations provide immediate feedback about the perceived novelty and attractiveness of the respective topics, of methods applied, and of the research findings offered.

My quick, subjective analysis of the issues addressed by the NOFOMA contributors-which, of course, does not claim not to meet standards of scientific rigour-suggests that 70 of the 86 papers could be assigned to just five topical clusters:

- 18 were explicitly dealing with aspects of sustainability in logistics and supply chain management, including topics of sustainable city logistics, energy conservation, and multi-modality.

P. Klaus $(\bowtie)$

Logistics Research, University Erlangen-Nuermberg,

Nuremberg, Germany

e-mail: peter.klaus@wiso.uni-erlangen.de

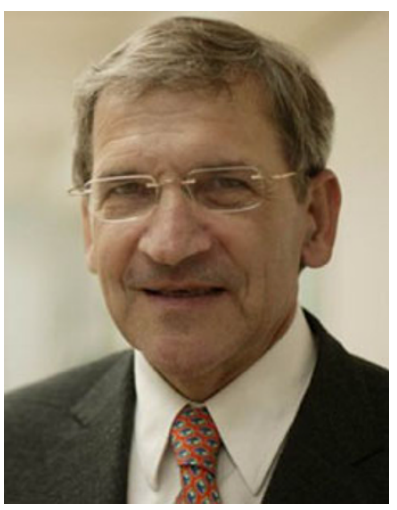

- Another 18 papers addressed issues of cooperation, collaboration, communication, relationships and organization between actors in supply networks.

- The third cluster of 17 papers focused on performance management-towards the "rationalization", "industrialization", application of new technologies-and performance measurement of logistical systems. A trend here seems to be the extension of this kind of research from the levels of individual enterprises and specific supply networks to the levels of national and international performance comparisons.

- A slightly smaller number of papers focused on issues of uncertainty, risk, and safety in the design and management of logistical systems, including contributions to the now popular topic of "humanitarian logistics".

- And last not least, there was a smaller cluster of papers around the questions of the appropriateness of certain research methods-especially the "case method"-for rigorous logistics research.

The BVL-conference, different from the open-content NOFOMA event, had

- "Coordinated Autonomous Systems"

as its guiding theme. Many contributions at BVL sessions revolved around the analogy of the "cloud" and "cloud computing". Cloud was discussed as a promise towards future logistics which would be suited to master the challenges of a sustainable economy, and the new demands of an "age of volatility".

If this snapshot summary of two logistics research conferences is interpreted correctly as reflecting current "Megatrends" in our economies and societies towards sustainability, economic performance new, and more 
intensive modes of individual, organizational, and societal cooperation, while dealing with increasing environmental uncertainty, shortage of certain resources, risks and volatility, then it may provide orientation to researchers in at least two ways: For one, it marks the current research mainstream. Next research projects within the mainstream should be drilling ever deeper, seek more differentiation and the application of still better data and methods to avoid repetition. And it shows where mainstream is not-where there are opportunities for innovative, non-conventional, out-of-the-box research efforts.

LOGISTICS RESEARCH welcomes contributions of both kinds-research which is deepening knowledge about familiar issues, and research on new topics-as this issue of our journal once again: Consider the provocative argument on the role of systems theory in logistics by Lindskog, and Bravo/Maim/Andrew's discussion of the future role of biofuel in our energy supply! And learn about advances in decision support research for the solution of complex inventory, vehicle routing, and location issues through the papers by Ramaekers/Janssens, Drexl, and Böttcher/ Schneider/Hackstein!.

We look forward to your comments on these papers, to more of your manuscript submissions, and your ideas for next year's special topic selections in LOGISTICS RESEARCH! Best regards,

Yours

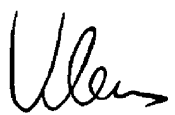

Peter Klaus

Editor-in-Chief LOGISTICS RESEARCH

June 2012 